\title{
Heating of magnetic fluid systems driven by circularly polarized magnetic field
}

\author{
Osman O. Ahsen ${ }^{a}$, Ugur Yilmaz ${ }^{a}$, M. Deniz Aksoy ${ }^{a}$, Gulay Ertas $^{b}$, Ergin $_{\text {Atalar }}{ }^{\mathrm{a}, *}$ \\ ${ }^{a}$ Department of Electrical and Electronics Engineering, Bilkent University, EE403 Bilkent, Ankara 06800, Turkey \\ ${ }^{\mathrm{b}}$ Department of Chemistry, Bilkent University, Ankara 06800, Turkey
}

\section{A R T I C L E I N F O}

\section{Article history:}

Received 13 March 2010

Received in revised form

3 May 2010

Available online 27 May 2010

Keywords:

Circular magnetic field

Ferrofluid

Heating

Magnetic fluid hyperthermia

Superparamagnetism

\begin{abstract}
A B S T R A C T
A theory is presented to calculate the heat dissipation of a magnetic suspension, a ferrofluid, driven by circularly polarized magnetic field. Theory is tested by in vitro experiments and it is shown that, regardless of the character of the relaxation process, linearly and circularly polarized magnetic field excitations, having the same root-mean-square magnitude, are equivalent in terms of heating efficiency.
\end{abstract}

(c) 2010 Elsevier B.V. All rights reserved.

\section{Introduction}

Hyperthermia, as a treatment of cancer, has recently attracted the attention of many researchers. Compared to chemotherapy and radiotherapy, it is expected that hyperthermia has fewer restrictive side effects and can also be used in combination with those therapies [1]. In contrast to many other hyperthermia techniques, magnetic fluid hyperthermia (MFH) has the advantage of selectively targeting the tumor tissue, so the risk of collateral organ damage is minimized [2]. In addition, focusing of the energy to a specific region is possible when radio frequency and static gradient field are used together [3]. MFH procedure starts with the injection of specifically synthesized superparamagnetic nanoparticles to the tumor site. When an external dynamic magnetic field is applied, induced magnetization lags the external field which results in heat dissipation at the target tissue.

There are several restrictions of the MFH procedure. Eddy currents, causing unwanted heating of tissue that is not targeted, limit the amplitude and the frequency of the applied field, i.e. one cannot apply arbitrarily large field amplitudes and frequencies. The extent of this issue is examined by Atkinson et al. [4] and it is concluded that the product of frequency and magnitude of the dynamic magnetic field should be less than a certain value. The second limitation is nerve stimulation threshold which similarly

\footnotetext{
* Corresponding author. Tel.: +90312 2903272; fax: +90312 2903001.

E-mail address: ergin@ee.bilkent.edu.tr (E. Atalar).

URL: http://www.bilkent.edu.tr (E. Atalar).
}

restricts the field strength and frequency [5]. Moreover, due to toxication effects, the amount and density of the injected magnetic fluid should be limited [6,7]. Therefore, it is essential to use the least magnetic field amplitude while obtaining the highest heating in the tumor tissue.

Several research studies have been conducted to achieve higher heating rates by optimizing the particle properties such as its diameter, shape and fraction or field properties such as its frequency and amplitude [8,9]. All of these studies assumed a linearly polarized magnetic field excitation and little research is done on the polarization of the magnetic field itself. Chatel et al. [10] calculated the heating rates of an isotropic magnetic fluid excited by a circularly polarized magnetic field. Their results indicated that for the low frequency regime of less than $1 \mathrm{MHz}$, circularly polarized magnetic field produces less power dissipation compared to linearly polarized field with the same field strength, given that the dominant relaxation mechanism is the Neel process. However, anisotropy of particles is an important property of relaxation mechanisms therefore significant for the heating dynamics. Recently, Cantillon-Murphy et al. [11] investigated the use of rotating magnetic fields in presence of a large DC field. They have shown with a numerical simulation that significant heating takes place, however, conditions such as field strength or particle size for which their results are valid were not studied.

In this study, heating properties of magnetic fluids containing superparamagnetic nanoparticles excited by a circularly polarized magnetic field is investigated. Power dissipation expression for the circularly polarized field excitation is presented and compared 
with the heating properties of the linearly polarized excitation to show that regardless of the character of the relaxation process, heating under linearly and circularly polarized magnetic fields is equal under conditions relevant to MFH, if the applied fields are equivalent in the root-mean-square (RMS) sense. Finally, theoretical predictions are tested by in vitro experiments.

\section{Theory}

To calculate the power dissipation due to arbitrarily applied magnetic field, the response of the magnetic medium, which is, in our case, a suspension containing fine magnetic particles, has to be known. The response of the magnetic fluid, $\vec{M}_{0}$, induced when static external magnetic field, $\vec{H}_{0}$, is applied, is well known. Following Langevin's treatment for paramagnetic systems, where the suspension is treated as a collection of non-interacting, thermally agitated magnetic dipoles, the magnitude of the equilibrium magnetization is $\vec{M}_{0}=\chi_{0} \vec{H}_{0}$ where equilibrium susceptibility, $\chi_{0}$, is given by [12]

$\chi_{0}=\chi_{i} \frac{3}{\xi}(\operatorname{coth} \xi-1 / \xi)$

with $\chi_{i}=\mu_{0} \phi M_{d}^{2} V_{m} / 3 k_{b} T$ and $\xi=\mu_{0} M_{d} V_{m} H_{0} / k_{b} T$, where $\mu_{0}$ is the permeability of the free space, $\phi$ is the volume concentration of the magnetic fluid, $M_{d}$ is the domain magnetization of the particle, $k_{b}$ is Boltzmann's constant, $T$ is the absolute temperature and $V_{m}$ is the magnetic volume of a particle. We remark that $\vec{M}_{0}$ is a vector quantity which is directed along $\vec{H}_{0}$ and $H_{0}$ represents the magnitude of $\vec{H}_{0}$.

For the dynamic case both the applied field, $\vec{H}(t)$, and the induced magnetization, $\vec{M}(t)$, depend on time. Shliomis introduced the dynamic equations of the induced magnetization of a magnetic fluid excited by time-varying magnetic field [13]. A ferrohydrodynamic Navier-Stokes equation and an equation relating averaged internal angular momentum with fluid vorticity describes the magnetic fluid motion. Supplied with a phenomenological equation of magnetization, these relations consistently describe the dynamics of a magnetic suspension. Under the assumption that excitation frequencies are much lower than the Larmor precession rate of the magnetic moments of the particles, it is possible to express the dependency of magnetization of the fluid with respect to fluid vorticity, and applied field, $\vec{H}(t)$. We assume that under conditions relevant to hyperthermia fluid vorticity is equal to zero, i.e. the fluid is essentially motionless. Therefore the governing equation of the dynamics of induced magnetization is given by [14]

$\frac{d \vec{M}(t)}{d t}=-\frac{\vec{M}(t)-\vec{M}_{e}(t)}{\tau}-\frac{\mu_{0}}{6 \phi \eta}(\vec{M}(t) \times(\vec{M}(t) \times \vec{H}(t)))$

where $\vec{M}_{e}(t)$ is the equilibrium magnetization given by $\chi_{0} \vec{H}(t), \tau$ is the characteristic time of the relaxation process, and $\eta$ is the viscosity of the carrier fluid. Other parameters were defined previously. Also note that (2) is given in the metric system, which is not the case in Shliomis's original paper [13]. $\tau$ in (2) is due to contributions of both Brownian and Neel relaxation processes. For $\tau_{b}=3 \eta V_{h} / k_{b} T$ and $\tau_{n}=\tau_{0} \exp \left(K V_{m} / k_{b} T\right)$, where $V_{h}$ is the hydrodynamic volume of the particle given by $V_{h}=(1+\delta / R)^{3} V_{m}, R$ is the core radius and $K$ is the anisotropy constant of the magnetic particle, $\delta$ is the thickness of the adsorbed surfactant layer, resultant characteristic time is $\tau=\left(1 / \tau_{b}+1 / \tau_{n}\right)^{-1}$, given that Brownian and Neel relaxation processes act in parallel [13].

The last term in (2), namely the cross-term, is due to non-zero internal angular momentum of the suspension. An alternating, linearly polarized magnetic field induces rotational swings of the grains but does not single out any preferred direction of their rotation. That is, an averaging over a small element of magnetic fluid volume results in internal angular momentum being identically zero, only if the excitation is linearly polarized [15]. However, under circularly polarized excitation this is not the case, i.e. internal angular momentum is different from zero, therefore, the effect of the cross-term has to be considered. In the following the consequences of ignoring the cross-term will be investigated, and then the conditions for which such an assumption holds true will be examined.

The assumption that cross-term is negligible results in a simple linear relaxation equation that is valid both for linearly and circularly polarized excitations. In such a case the time dependent evolution of the induced magnetization can be expressed in terms of equilibrium magnetization $\vec{M}_{e}(\mathrm{t})$, therefore in terms of applied field $\vec{H}(t)$ :

$\frac{d \vec{M}(t)}{d t}=-\frac{\vec{M}(t)-\vec{M}_{e}(t)}{\tau}$

This is the common formulation of the relaxation process of the induced magnetization. It is widely accepted in the literature [12] and leads to a simple expression and the understanding of the dissipation mechanism.

In the case of a circularly polarized excitation such that $\vec{H}_{c}(t)$ is rotating on $x-y$ plane with an RMS magnitude of $H_{0} / \sqrt{2}$, we have $\vec{H}_{c}(t)=\mathfrak{R}\left(H_{0} \hat{a}_{+} e^{j \omega t}\right)$. Notice that $\hat{a}_{+}$is unit rotating vector and is given in terms of cartesian unit vectors as $\left(\hat{a}_{x}+j \hat{a}_{y}\right) / \sqrt{2}$. Here $\mathfrak{R}(\cdot)$ implies the real component of the expression. Hence the phasor equivalent of the applied field $\vec{H}_{c}$ will be given by $H_{0} \hat{a}_{+}$. On the other hand, linearly polarized excitation with the same RMS magnitude is given by $\overrightarrow{H_{l}}(t)=\Re\left\{H_{0} e^{j \omega t} \hat{a}_{x}\right\}$, with $H_{0} \hat{a}_{x}$ being the phasor equivalent. Equilibrium magnetization(s) are $\vec{M}_{c e}=$ $\chi_{0} H_{0} \hat{a}_{+}$and $\vec{M}_{l e}=\chi_{0} H_{0} \hat{a}_{x}$ for the circularly and the linearly polarized excitations, respectively. Plugging $\vec{M}_{c e}$ and $\vec{M}_{l e}$ into the phasor representation of (3), $j \omega \vec{M}=-\left(\vec{M}-\vec{M}_{e}\right) / \tau$, and solving for induced magnetizations $\vec{M}_{c}$ and $\vec{M}_{l}$ yields

$\vec{M}_{c}=\frac{1}{\sqrt{1+\tau^{2} \omega^{2}}} \chi_{0} H_{0} e^{-j \tan ^{-1}(\omega \tau)} \hat{a}_{+}$

$\vec{M}_{l}=\frac{1}{\sqrt{1+\tau^{2} \omega^{2}}} \chi_{0} H_{0} e^{-j \tan ^{-1}(\omega \tau)} \hat{a}_{x}$

Note that $\vec{M}_{c}$ is also rotating on $x-y$ plane and lags $\vec{H}_{c}$ by $\tan ^{-1}(\omega \tau)$ radians. For large $\omega \tau$, the angle between $\vec{H}_{c}$ and $\vec{M}_{c}$ increases, however, magnitude of $\vec{M}_{c}$ drops proportional to $1 / \omega \tau$. Notice that, similar to circularly polarized excitation, magnetization lags the linearly polarized excitation by $\tan ^{-1}(\omega \tau)$ radians.

Once this phase lag between the excitation and the induced magnetization is known, calculating the power dissipation for both excitation modalities is straightforward using the power expression $P=\mathfrak{R}\left\{\frac{1}{2} j \omega \mu_{0} \vec{H} \cdot \vec{M}^{*}\right\}$. Plugging in phasor representations of the applied field, $\vec{H}_{c}$ and $\vec{H}_{l}$, and the corresponding expressions for induced magnetization, $\vec{M}_{c}$ and $\vec{M}_{l}$, into this expression we arrive at the power dissipation expressions for a mono-disperse magnetic fluid. Resulting expressions are identical for linearly and circularly polarized excitation modalities and given by

$P=\frac{1}{2} \mu_{0} \chi_{0} H_{0}^{2} \omega \frac{\omega \tau}{1+(\omega \tau)^{2}}$

Eq. (6) implies that circularly and linearly polarized excitations result in identical power dissipation if the RMS magnitudes of the magnetic fields of the two excitation modalities are the same.

For the circularly polarized excitation, the applicability of the power dissipation formula in (6) depends on the assumption that the cross-term in (2) is negligible. There are two forcing terms on the right side of (2): the linear term and the latter cross term. The 
magnitudes of these two terms will be compared for the steadystate solution of $\vec{M}_{c}$ in (4). A straightforward phasor analysis yields the magnitude of the cross term as

$\left|\frac{\mu_{0}}{6 \phi \eta} \vec{M}_{c} \times\left(\vec{M}_{c} \times \vec{H}_{c}\right)\right|=\frac{\mu_{0} \chi_{0}^{2} H_{0}^{3} \omega \tau}{6 \phi \eta\left(1+\omega^{2} \tau^{2}\right)^{3 / 2}}$

On the other hand, the magnitude of the linear term is given by

$\left|\frac{\vec{M}_{c}-\vec{M}_{c e}}{\tau}\right|=\frac{H_{0} \chi_{0} \omega}{\sqrt{1+\omega^{2} \tau^{2}}}$

Therefore, if the cross term in (7) is much smaller than the linear term in (8), then the cross-term is negligible. This implies

$H_{0} \ll \sqrt{\frac{6 \phi \eta\left(1+\omega^{2} \tau^{2}\right)}{\mu_{0} \chi_{0} \tau}}$

The right hand side of the inequality above is the upper bound for the field strength below which the governing equation of the dynamics of magnetization for the circularly polarized excitation is given by (3).

In a practical MFH process, the magnitude of the applied field is restricted by the limitations of several safety concerns. According to Atkinson et al. [4], to prevent excess eddy current heating multiplication of frequency and magnitude of the dynamic magnetic field should be less than a specific value given by

$H_{0} \leq \frac{4.85 \times 10^{8} \mathrm{~A} / \mathrm{m} \mathrm{s}}{f}$

Another limitation for the field strength is related to the peripheral nerve stimulation threshold. The following equation is commonly used to prevent nerve stimulation under an alternating magnetic field [5]:

$H_{0} \leq \frac{1.07 \times 10^{7} \mathrm{~A} / \mathrm{m} \mathrm{s}}{f}+5.93 \times 10^{3} \mathrm{~A} / \mathrm{m}$

For a given particle radius, therefore a particular relaxation time, there exists an optimum excitation frequency, $\omega_{o p t}$, and a corresponding maximum allowable field strength, $H_{\max }$, such that power dissipation expression (6) is maximized while satisfying inequalities (10) and (11) hence ensuring patient safety. As an example, for a water based mono-disperse magnetic fluid with particle radius of $10 \mathrm{~nm}$, power dissipation given by (6) is plotted with respect to frequency in Fig. 1, showing that power dissipation is maximized at a specific excitation frequency.

We assume that in a practical application of MFH excitation frequencies will be around $\omega_{\text {opt }}$. We then solve (1) and (9) simultaneously to find the upper bound of the field strength, which we call $H_{\text {upper }}$. Fig. 2 illustrates the relation between particle radius $(R)$ and the upper bound of the field strength $\left(H_{\text {upper }}\right)$, below which the cross-term in (2) is negligible. The maximum allowable field strength $\left(H_{\max }\right)$ for the patient safety is also plotted. Here, for a worst case scenario, we take $\eta=0.001 \mathrm{~kg} / \mathrm{ms}$ for a water based suspension and $\eta=0.005 \mathrm{~kg} / \mathrm{ms}$ for blood. Volume concentration is assumed on the same graph to be $\phi=0.1$ and domain magnetization of the suspended particle is $M_{d}=400 \mathrm{kA} / \mathrm{m}$.

In Fig. 2 we see that for a water based magnetic fluid, the upper bound of the field strength below which the cross-term becomes negligible is around $20 \mathrm{kA} / \mathrm{m}$. However, patient safety limits already restricts the field strength below $10 \mathrm{kA} / \mathrm{m}$. Moreover, as the viscosity increases towards conditions of the human tissue, the upper bound also increases much rapid than the safety limit.

As a result, theory suggests that for the Neel regime, and as well as for the Brownian regime, if the patient safety limits are taken into consideration such that excitation frequency and the field strength are restricted within a region for which a more general equation of magnetization (2) reduces into simple linear relaxation equation (3) and the two excitation modalities, linearly and circularly polarized excitations, become equivalent in terms of heating efficiency. This whole analysis is based on numerous assumptions such as the validity of (2), magnetic fluid being motionless and mono-dispersity of the particles, therefore it was necessary to conduct experiments in order to verify the predictions of the theory.

\section{Experiments}

A schematic for the experimental setup is given in Fig. 3.

In order to generate a rotating magnetic field, four solenoids are used: two of them facing each other, creating a linear magnetic field in $x$ direction; and the other two in $y$ direction, creating the orthogonal magnetic field component. Solenoids are manufactured in conic shape, and the dimensions are optimized using a field calculator to maximize the magnetic field strength at the region of interest. The dimensions can be seen from Fig. 4.

Litz wire is used to reduce the proximity effect. Each solenoid has around 150 turns and three layers, having 50 turns in each layer. Two solenoids are connected and placed in $x$ direction, while the other two solenoids are connected and placed in $y$ direction.

The mutual inductance between pairs of inductors is minimized by applying current to one pair, and measuring the voltage induced on the other pair with an oscilloscope (Tektonix ${ }^{\mathrm{TM}}$ TDS2024). The second pair is then slowly rotated around $z$ axis, until the measured voltage reaches to a minimum. Then the

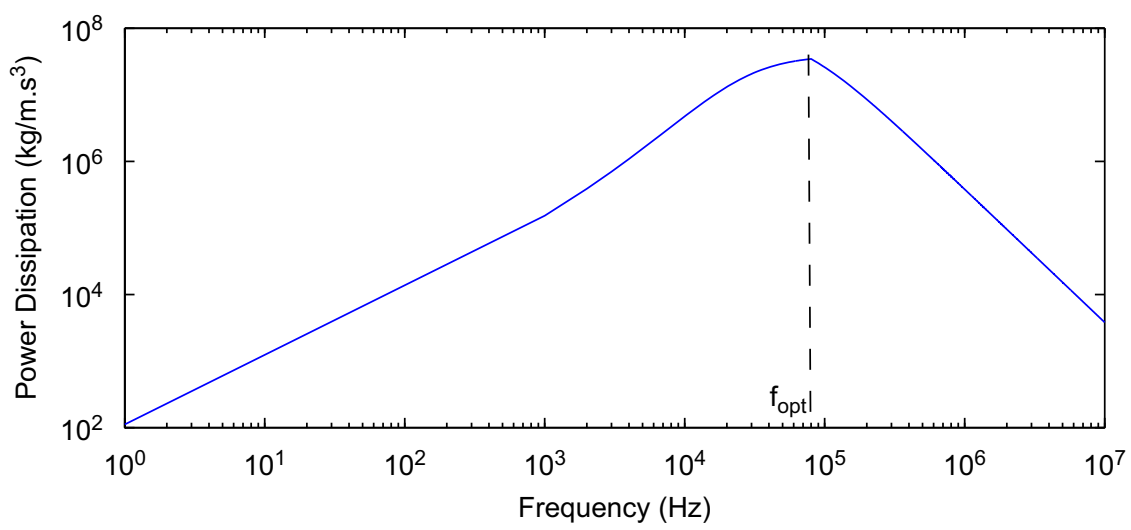

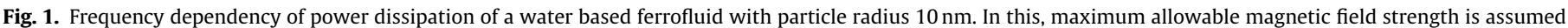
such that patient safety thresholds are not exceeded. The optimum frequency is around $80 \mathrm{kHz}$ for this particle size. 


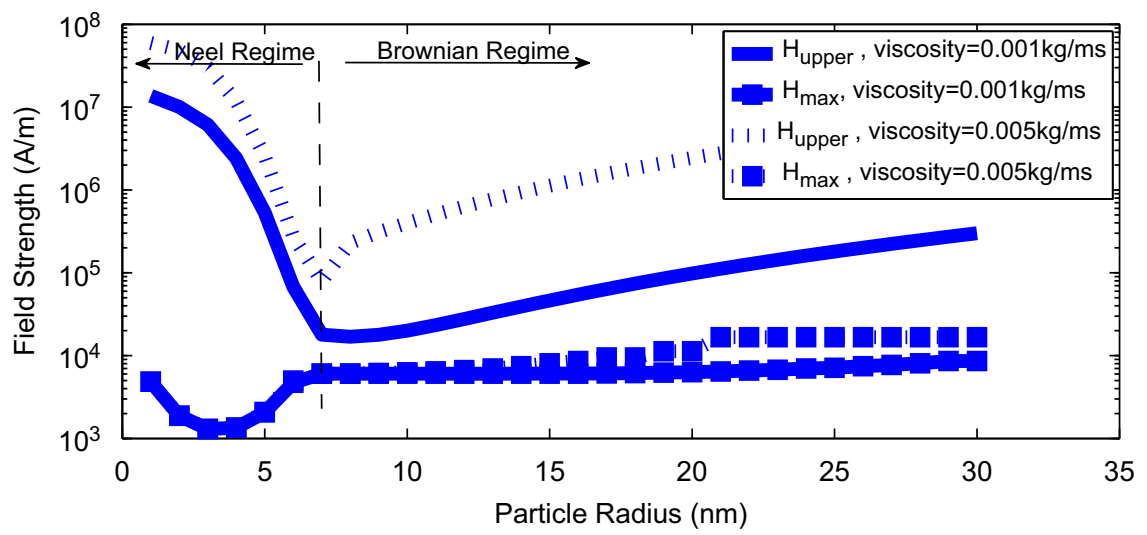

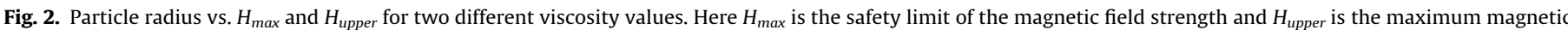
field strength such that the dynamics of the magnetic suspension under circularly polarized magnetic field can be formulated by a linear relaxation equation.

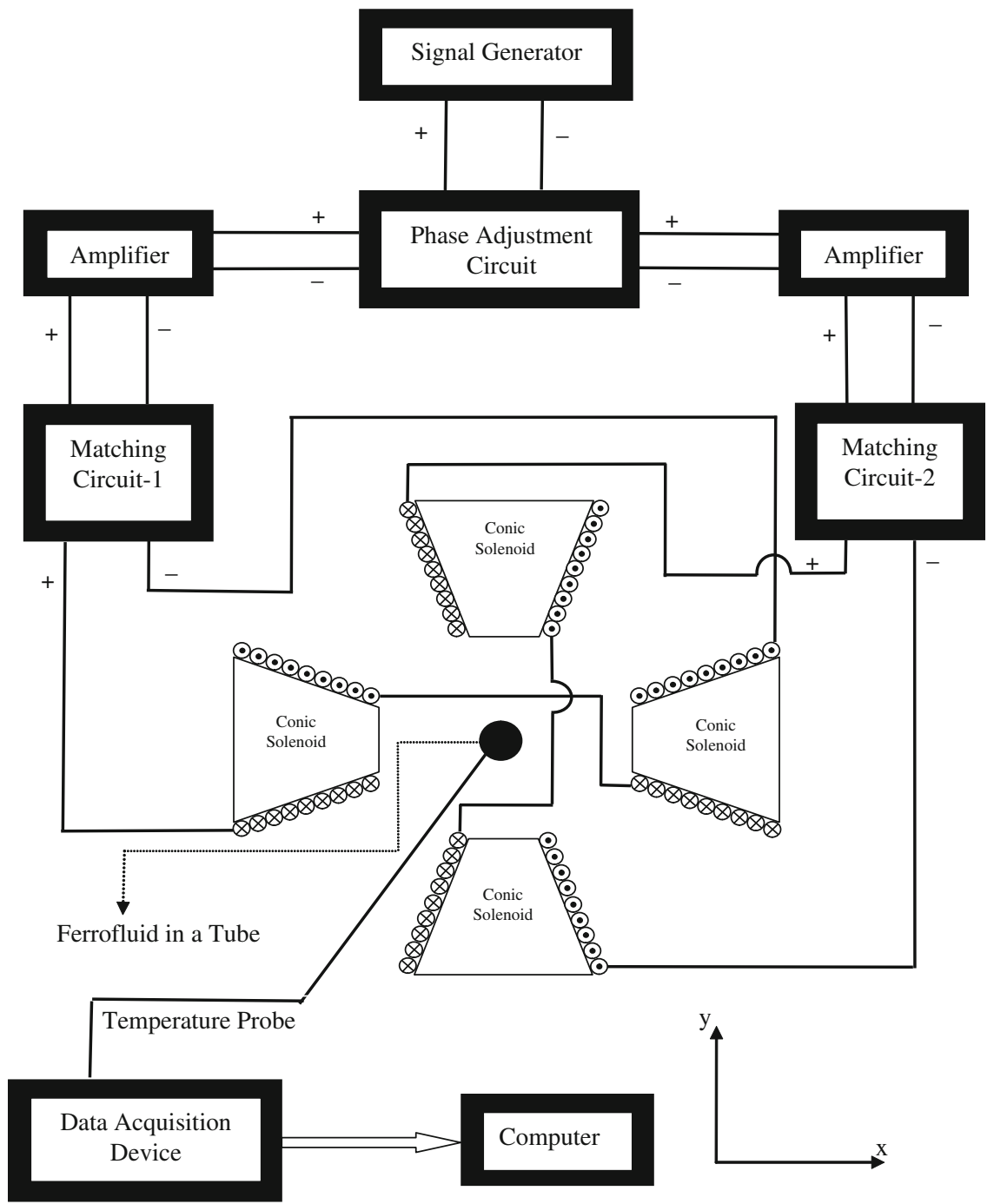

Fig. 3. The general schema of the experimental setup. Using this setup circular and linear magnetic fields can be obtained.

solenoids are fixed to a board to ensure a stable experiment environment.

For each frequency, impedance matching is done with the help of a network analyzer $\mathrm{HP}^{\mathrm{TM}} 8753 \mathrm{D}$ between the solenoid pairs and the power amplifier Tonylee ${ }^{\mathrm{TM}} \mathrm{DJ} 451$ having $8 \Omega$ of output impedance. Further, to create a circularly polarized magnetic field, an RC circuit is used to ensure that the orthogonal components of the magnetic field have $90^{\circ}$ of phase shift in between and have the same field strength. The phase shift and the magnitude of the magnetic field components at the region of interest are measured using a pickup coil of 5 turns which is connected to an oscilloscope. Pickup coil is rotated, keeping the position of the 


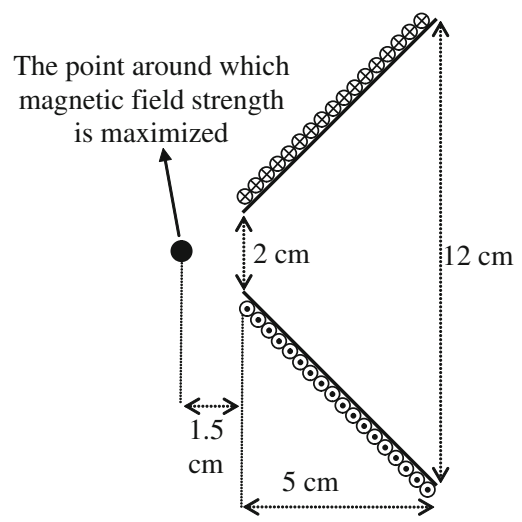

Fig. 4. Dimensions of solenoids used in the experiments.

coil fixed in the middle of four solenoids, and the voltage is measured at all directions. By adjusting phase and amplitude of the magnetic fields at two main directions using variable resistors and amplification factors of the amplifiers, a circularly polarized magnetic field is obtained.

A test tube of $1 \mathrm{~cm}$ diameter filled with magnetic fluid sample is placed in the middle of the solenoids. A fiber optic temperature probe is placed into the fluid and temperature increase is recorded using a data acquisition device with the source data coming from Neoptix ${ }^{\mathrm{TM}}$ temperature sensors, which samples data at $0.3 \mathrm{~s}$ time intervals with $0.1^{\circ} \mathrm{C}$ temperature precision. Two different commercially available magnetic fluid samples are used in the experiments: a water based (Liquids Research ${ }^{\mathrm{TM}}$, WHKS1S9-C) and an oil based (Liquids Research ${ }^{\mathrm{TM}}$, WHJS1-C) suspension. Average diameter of nanoparticles is reported to be less than $10 \mathrm{~nm}$, saturation magnetization (Ms) to be around $500 \mathrm{G}$ and ferrite concentration to be around $10 \%$. In order to determine shape and dimensions of nanoparticles a TEM with model Tecnai ${ }^{\mathrm{TM}} \mathrm{G}$ F30 is used. The particles are coated with carboxylic acid based surfactant which is chosen to be compatible with the solvent to prevent the agglomeration of the particles in polar solvent.

To ensure the reliability of the experiments, a repeatability experiment is conducted both for circularly and linearly polarized excitations. Experiments are repeated six times and the variation of the slope of heating is observed. Thermal isolation of the experimental setup is tested by an experiment conducted using a water filled test tube.

Although a vast range of frequencies and amplitudes have been used in hyperthermia applications, we chose our experimental parameters by taking the nerve stimulation and eddy current thresholds into account, and chose an excitation frequency around $50 \mathrm{kHz}$. It is also illustrated recently that for a mean particle diameter around $8 \mathrm{~nm}$, excitation frequency of $80 \mathrm{kHz}$ yields an optimal heating efficiency [9]. Therefore, experiments are conducted for frequencies of 60 and $70 \mathrm{kHz}$. Amplitude of the orthogonal components of the field was $1.8 \mathrm{kA} /$ $\mathrm{m}$ for circularly polarized excitation. On the other hand, field amplitude was $2.55 \mathrm{kA} / \mathrm{m}$ for linearly polarized excitation, i.e. circularly and linearly polarized fields were equivalent in the RMS sense. For completeness, further experiments for linearly polarized excitation is repeated for a field amplitude of $1.8 \mathrm{kA} / \mathrm{m}$.

To determine the dominant relaxation mechanism of the particles, the experiments were also conducted by suspending the water based magnetic fluid in a commercial gel. Therefore, Brownian relaxation of the particles is inhibited. Further, the water based fluid is diluted to an amount that it has the same concentration as the suspended particles in gel. As a result both samples had about $6 \%$ of ferrite concentration. Circularly polarized magnetic field with an RMS magnitude of $2 \mathrm{kA} / \mathrm{m}$ is used for both magnetic particles suspended in gel and water based magnetic fluid having the same particle concentration. Heating slopes of the two experiments are compared.

\section{Results and discussion}

The results of the repeatability experiment indicated that the slope of the time vs. temperature curve varies at most $0.2^{\circ} \mathrm{C} / \mathrm{min}$ from its mean value with a standard deviation of $0.14^{\circ} \mathrm{C} / \mathrm{min}$. In addition, it is observed that the temperature of the water sample stays constant (variation is less than the precision of the censor, $0.1^{\circ} \mathrm{C} / \mathrm{min}$ ) revealing that heat generated in the coil winding is not a significant error source. As a sample case, heating experiment results of the oil based ferrofluid at $60 \mathrm{kHz}$ for a circularly polarized field excitation of RMS magnitude $1.8 \mathrm{kA} / \mathrm{m}$ is shown in Fig. 5. The fluctuations are due to the quantization error of the data acquisition device.

Thick line in Fig. 5 represents a linear fit to a collection of six subsequently acquired data belonging to a single experiment. Based on this approach the heating slope of each different experiment is determined and the corresponding standard deviation of the slope is calculated. Finally well known statistical procedure, ANOVA (analysis of variance), is applied, and based on the null hypothesis that linearly and circularly polarized magnetic fields having the same RMS magnitude result in the same amount of heating, corresponding $F$ values are calculated. Results are given in Table 1.

According to the $F$-distribution table, the critical $F$ value for a significance level of $5 \%$ is $F_{0.05 ; 1,4}=7.71$, where the subscript 1 denotes the degrees of freedom for treatments and subscript 4 denotes the degrees of freedom for the error. The fact that the $F$ values of the experiment results, given in Table 1 , are much less than this critical value shows that these results do not contradict the initial hypothesis. This indicates that linearly and circularly polarized magnetic fields having the same RMS magnitude are equivalent in terms of heating efficiency regardless of the excitation frequency and the carrier liquid used. Furthermore, it is observed that linearly polarized field with RMS magnitude of $1.8 \mathrm{kA} / \mathrm{m}$ results in two times the heat dissipation compared to linearly polarized field with RMS magnitude of $1.27 \mathrm{kA} / \mathrm{m}$, which agrees with the fact that power dissipation is proportional to the square of the magnetic field strength as is predicted by the general power dissipation equation of (6).

The average heating slopes of the experiments with magnetic particles suspended in gel and water based magnetic fluid having the same particle concentration were observed as 0.41 and $0.48^{\circ} \mathrm{C} / \mathrm{min}$, respectively. The small difference of the heating slopes of the two samples indicates that under circularly polarized magnetic field Neel process dominates the relaxation mechanism of the magnetic particles having mean diameter around $10 \mathrm{~nm}$. Formerly Wang et al. [16] conducted a similar experiment to show that also for linearly polarized magnetic field Neel process is dominant relaxation mechanism for particle diameters up to $10 \mathrm{~nm}$. This, along with previous discussions, confirms our analysis that circularly polarized field excitation does not produce less power dissipation for the frequencies relevant to magnetic fluid hyperthermia, even if the dominant relaxation mechanism is the Neel process.

It is known that agglomeration significantly reduces the heating in MFH [16]. However, even if the sample is agglomerated to a certain extent, agglomerates will not contribute to the heating, since for the field strengths related to hyperthermia hysteresis losses are insignificant [17]. Therefore, we can say that possible agglomerations do not change mechanism of relaxation and do not effect the validity of our conclusions. 


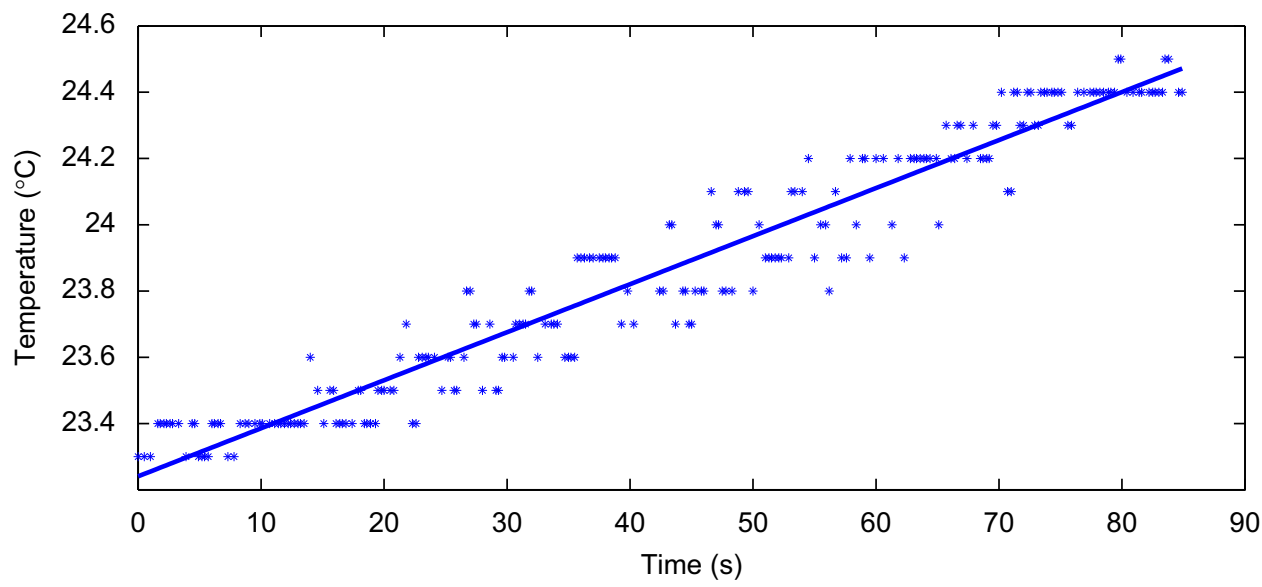

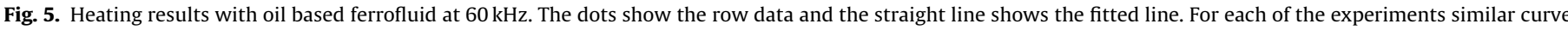
fitting was conducted and the slopes of these lines are used in the results.

Table 1

Experiment results.

\begin{tabular}{|c|c|c|c|c|c|}
\hline $\begin{array}{l}\text { Base of the } \\
\text { sample }\end{array}$ & $\begin{array}{l}\text { Frequency } \\
(\mathrm{kHz})\end{array}$ & $\begin{array}{l}\text { Heating with linear field of RMS } \\
\text { amplitude }(1.27 \mathrm{kA} / \mathrm{m})\left({ }^{\circ} \mathrm{C} / \mathrm{min}\right)\end{array}$ & $\begin{array}{l}\text { Heating with linear field of RMS } \\
\text { amplitude }(1.8 \mathrm{kA} / \mathrm{m})\left({ }^{\circ} \mathrm{C} / \mathrm{min}\right)\end{array}$ & $\begin{array}{l}\text { Heating with circular field of RMS } \\
\text { amplitude }(1.8 \mathrm{kA} / \mathrm{m})\left({ }^{\circ} \mathrm{C} / \mathrm{min}\right)\end{array}$ & $F$-Value \\
\hline Oil & 60 & $0.468 \pm 0.08$ & $0.768 \pm 0.08$ & $0.87 \pm 0.12$ & 1.05 \\
\hline Oil & 70 & $0.516 \pm 0.07$ & $1.08 \pm 0.07$ & $0.97 \pm 0.1$ & 1.61 \\
\hline Water & 60 & $0.18 \pm 0.05$ & $0.414 \pm 0.07$ & $0.348 \pm 0.05$ & 0.82 \\
\hline Water & 70 & $0.26 \pm 0.06$ & $0.468 \pm 0.06$ & $0.522 \pm 0.07$ & 0.88 \\
\hline
\end{tabular}

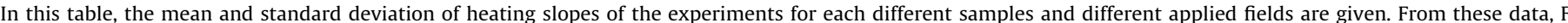

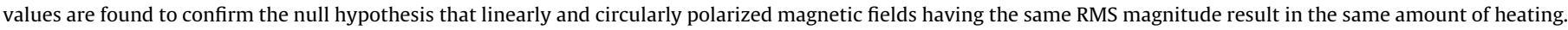

It should be noted that it is still possible for large field amplitudes or large particle diameters that circularly and linearly polarized field excitations could result in different heating rates. As the cross-term in (2) becomes significant, heating rates under circularly polarized excitation will decrease. However, if the aforementioned safety limitations are considered, such large field amplitudes, for which the two excitation modalities start behaving differently, are unlikely to be used in a usual MFH application.

It is possible to put (2) into test by exciting a magnetic fluid with a circularly polarized magnetic field strong enough that the cross-terms starts to dominate the relaxation process. Such an experiment could verify the validity of (2), and hence the validity of the fundamental understanding of the dynamics of a magnetic fluid excited by a time varying magnetic field. However, since the required field strength is unusually high for a practical application of MFH treatment, we have not carried out such an experiment.

\section{Conclusion}

A theory is presented to calculate heating of a magnetic suspension driven by circularly polarized magnetic field. It has been shown that regardless of the character of the dominant relaxation process, linearly and circularly polarized magnetic field excitations, having the same root-mean-square magnitude, are equivalent in terms of heating efficiency. This theory was verified by in vitro experiments.

\section{Acknowledgments}

We are very grateful to M.I. Shliomis from Ben-Gurion University of the Negev for his helpful discussions on the subject,
UNAM-Institute of Materials Science and Nanotechnology for providing the TEM for the visualization of the nanoparticles, Burak Akin of Bilkent University Electrical and Electronics Engineering Department for his help on the preparation of the experimental setup.

\section{References}

[1] P. Moroz, S.K. Jones, B.N. Gray, Magnetically mediated hyperthermia: current status and future directions, International Journal of Hyperthermia 18 (4) (2002) 267-284

[2] S. Mornet, S. Vasseur, F. Grasset, E. Duguet, Magnetic nanoparticle design for medical diagnosis and therapy, Journal of Materials Chemistry 14 (14) (2004) 2161-2175.

[3] T.O. Tasci, I. Vargel, A. Arat, E. Guzel, P. Korkusuz, E. Atalar, Focused rf hyperthermia using magnetic fluids, Medical Physics 36 (2009) 1906-1912.

[4] W.J. Atkinson, I.A. Brezovich, D.P. Chakraborty, Usable frequencies in hyperthermia with thermal seeds, IEEE Transactions on BioMedical Engineering BME-31 (1) (1984) 70-75.

[5] D.J. Schaefer, J.D. Bourland, J.A. Nyenhuis, Review of patient safety in time-varying gradient fields, Journal of Magnetic Resonance Imaging 12 (2000) 20-29.

[6] Y.-G. Lv, Z.-S. Deng, J. Liu, 3-d numerical study on the induced heating effects of embedded micro/nanoparticles on human body subject to external medical electromagnetic field, IEEE Transactions on Nanobioscience 4 (4) (2005) 284-294.

[7] Q.A. Pankhurst, J. Connolly, S.K. Jones, J. Dobson, Applications of magnetic nanoparticles in biomedicine, Journal of Physics D-Applied Physics 36 (2003) R167-R181.

[8] G. Glockl, R. Hergt, M. Zeisberger, S. Dutz, S. Nagel, W. Weitschies, The effect of field parameters, nanoparticle properties and immobilization on the specific heating power in magnetic particle hyperthermia, Journal of Physics-Condensed Matter 18 (2006) S2935-S2949.

[9] T.O. Tasci, Focused RF ablation using magnetic fluids, Ph.D. Thesis, Bilkent University, August 2006

[10] P.F. de Chatel, I. Nandori, J. Hakl, S. Meszaros, K. Vad, Magnetic particle hyperthermia: neel relaxation in magnetic nanoparticles under circularly polarized field, Journal of Physics_Condensed Matter 21 (2009) 124202.

[11] P. Cantillon-Murphy, L.L. Wald, E. Adalsteinsson, M. Zahn, Heating in the MRI environment due to superparamagnetic fluid suspensions in a rotating 
magnetic field, Journal of Magnetism and Magnetic Materials 322 (2010) 727-733.

[12] R.E. Rosensweig, Heating magnetic fluid with alternating magnetic field, Journal of Magnetism And Magnetic Materials 252 (2002) 370-374.

[13] M.I. Shliomis, Magnetic fluids, Uspekhi Fizicheskikh Nauk 112 (1974) 427-458 (Translated by W. F. Brown, Jr).

[14] M.I. Shliomis, Ferrohydrodynamics: testing a third magnetization equation, Physical Review E 64 (2001) 060501.
[15] M.I. Shliomis, Ferrohydrodynamics: retrospective and issues, in: Lecture Notes in Physics, Springer-Verlag, Berlin, 2002, pp. 85-110.

[16] X.M. Wang, H.C. Gu, Z.Q. Yang, The heating effect of magnetic fluids in an alternating magnetic field, Journal of Magnetism and Magnetic Materials 293 (2005) 334-340.

[17] S. Dutz, R. Hergt, J. Mürbe, R. Müller, M. Zeisberger, W. Andrä, J. Töpfer, M.E. Bellemann, Hysteresis losses of magnetic nanoparticle powders in the single domain size range, Journal of Magnetism and Magnetic Materials 308 (2007) 305-312. 